\title{
Severe Performance Effects of Combined Jamming Interferences and Rayleigh Fading with Mitigation using Joint Complex Spreading and MIMO
}

\author{
Ric A. Romero*, Tri T. Ha \\ Electrical and Computer Engineering Department, Naval Postgraduate School, Monterey, CA, United States \\ *Corresponding Author: rnromero@nps.edu
}

Copyright (C2013 Horizon Research Publishing All rights reserved.

\begin{abstract}
In communications systems under jamming interference, it is customary to employ spread spectrum techniques to combat performance degradation. In systems with fading, spatial diversity techniques such as MIMO schemes are usually used to mitigate the problem. Here, we analyze and show the extremely severe effects on BER performance of the combined jamming signals and Rayleigh fading of a system undergoing both conditions at the same time. Then, we analyze and derive the performance of systems employing joint MIMO and complex spreading schemes to combat both Rayleigh fading and jamming and note the significant mitigation improvements.
\end{abstract}

Keywords Complex spreading, MIMO, Rayleigh fading, Barrage noise jamming, Pulsed noise jamming, Tone jamming, Maximal Ratio Combining (MRC)

\section{Introduction}

\section{$1.1 \quad$ Background}

It is well known that performance in terms of bit error rate (BER) of a conventional communication system degrades severely in a fading environment. Receiver diversity combining is used to ameliorate the effects. Also, spatial diversity benefits are now very well known [1-2]. Recently, both transmit and receive spatial diversities in terms of multiple-output-multiple-input (MIMO) techniques have shown to improve performances over fading channels. In terms of channel capacity, certain references that address capacity in various fading possibilities include (but certainly not limited to) [3-8]. The works in [9-10] introduced the idea of transmit (space-time) coding, which has flourished ever since. Some references include (but not limited to) [11-14]. In military communication systems, external interferences called jamming are practical problems. Jamming is often mitigated by the use of spread spectrum (SS) techniques. Spread spectrum technique as applied in both military for anti-jam and civilian systems for multi-user access such as code-division multiple access (CDMA) has a long history dating back from the famed actress Ms. Hedy Lamarr, who along with a collaborator submitted the idea of SS in 1941. In particular, the SS proposed was a frequency-hopping $(\mathrm{FH})$ technique as opposed to direct-sequence (DS) techniques. SS as a mitigation technique against jamming is a mature topic. As such it is not possible to list every major contribution but the interested reader is referred to some excellent references from early to more recent works such as [15-23].

\subsection{Motivation and Contributions}

Our focus in this paper is when a system experiences combined jamming interference and fading at the same time. It will be shown that effects of both jamming and fading on the BER performances are extremely severe compared to jamming or fading alone. While it is very intuitive to use joint spread spectrum and MIMO technique to combat such a combined problem, there is very little in the literature that show the extreme severity of the performance degradation when both effects are present and how much improvement in performance such a joint scheme would produce. In addition, most works in spread spectrum use traditional dual-channel SS which emphasizes the use of pseudo-noise (PN) codes. Dual-channel SS uses separate low-correlation PN codes on the I and Q channels. 
Unfortunately, this technique still exhibits performance loss known as spreading loss due to the low but nonetheless performance-degrading cross-talk issue that is present in the resulting I and Q data.

Here, we utilize complex spreading which does not induce spreading loss. Moreover, there's a need for BER expressions when both MIMO and SS are applied, in which designers can use to perform proper trade-offs when implementing practical systems. In this paper, it is our goal to fill these needs. 'True' complex spreading, where I-Q spreading is performed in the transmitter and the corresponding de-spreading happens in the receiver is mentioned in [24] but in that work, data bits are only utilized in the Q-channel and pilot is used in the I-channel for the purposes of channel estimation. In [25], complex spreading is applied to I-Q symbols to be modulated. The works in [26-27] concentrates on employing processing methods that permit the receiver to benefit from the correlation properties of complex sequences for quartenary phase shift keying (QPSK) modulations. In this paper, we utilize complex spreading which is shown to have no cross-talk problem and applies to all I-Q modulations including quadrature amplitude modulation (QAM). Thus, in this paper our goal is to derive, report, and evaluate BER for systems employing antenna diversity and complex spread-spectrum in Rayleigh fading and various jamming signals. Our own preliminary work covering this subject is reported in [28].

The humble contributions of the paper are as follows: a) we analyze the use of complex spreading and show that there is no cross-talk between I and Q channels which is present in the classical dual-channel spreading; b) we derive the covariances of three common jamming signals via signal-space vector formulation (which is usually performed in continuous-time) and incorporate the results to performance evaluation; c) we evaluate the severity of performance degradation of combined fading and jamming and we present the much needed SNR-related expressions for deriving BER curves for a system employing joint complex spreading and MIMO in combined fading and jamming (where BER becomes a function of various parameters of interest such as number of chips, signal-to-jamming ratio, jamming duty cycle, number of transmit antennas, MIMO diversity, etc.) that can be used by engineers to perform system trade-offs; and d) finally, although our formulation can be applied to various modulations, we derive and plot BER curves for 16-QAM to illustrate the mitigation effects of joint complex spreading and MIMO for systems under combined jamming and fading. A possible extension to this paper in order to boost performance is to include beamsteering (steerable antennas) along with the joint complex-MIMO schemes.

This work is outlined as follows. Section II provides the signal model background necessary to present this work which includes discrete-time formulation, jamming, fading, maximal ratio combining (MRC), orthogonal space-time block codes (OSTBC), and a generalized BER expression for fading environments only. The expression will be eventually modified such that the three various jamming types are accommodated and the mitigating effects of complex spreading are added to diversity combining. Section III discusses complex spreading, complex de-spreading, and detection. In Section IV, we derive the noise and interference covariances needed for the BER expressions. In Section V, we present results and use 16QAM to illustrate performance trade-offs. Finally, we present our conclusions in Section VI.

\section{Signal Modeling}

\subsection{Discrete-Time Formulation}

In traditional analysis of communication systems under jamming conditions, the continuous time model is extensively used in deriving performance metrics such as probability of bit error or BER. In modern designs, the signal processing of sampled signals lend itself well for signal-space vector analysis. In this paper, we utilize the discrete-time approach to model complex spreading MIMO systems in Rayleigh fading and interference.

\subsection{Jamming and Fading}

We consider Rayleigh fading channel and three different types of interference: broadband noise jamming (also known as barrage noise jamming), pulsed noise jamming, and tone jamming. Maximal ratio combining (MRC) [29-31], which is a combining technique that ensures maximum signal-to-noise ratio (SNR) is used. Perfect channel estimation is assumed. Orthogonal space-time block codes (OSTBC), such as Alamouti OSTBC [10], are used for different transmit diversities. In terms of modulation, the work here can be applied for various phase and quadrature amplitude modulations. We will use 16QAM for our example, illustration, and performance tradeoff discussions. We will analyze various diversity combinations to determine effective transmit/receive antenna combinations for the three types of jamming in a Rayleigh fading channel. The comparison assumes fixed signal bandwidth and fixed transmit power. We will see that for most cases broadband jamming is more effective than tone jamming. In pulsed jamming, the duty cycle is given by $\rho$. We will report the effect of increasing $\rho$ on BER as a function of signal-to-jam ratio.

The multipath fading channel is a commonly used model in wireless communications. Each signal path results in a randomly delayed, attenuated, and phase-shifted copy of the transmitted signal. These multipath copies combine at the receiver, where the received signal can be modeled as a Rayleigh fading process (for no line-of-sight path), a Rician fading process (for one line-of-sight path), or a Nakagami fading process. In addition, because the arrival times of the multipath copies are random, the multipath copies in some cases overlap the next symbol and cause inter-symbol interference. A channel is defined as flat fading when the multipath delay spread is less than the 
symbol time [25], [32]. For a flat fading channel, we let the complex channel tap coefficient be $h=|h| e^{j \theta}$, where $h$ is the attenuation coefficient of the signal and $\theta$ is the phase shift that the fading channel introduces.

\subsection{Maximal Ratio Combining}

As part of the detector, we will utilize the technique called maximal ratio combining (MRC). MRC is usually used for fading channels albeit without the presence of jamming and spreading. The addition of jamming and spreading will presented in Section III. For an $L$-fold diversity system, an optimum coherent combiner in the receiver must execute a combining operation in order to obtain a signal-to-noise ratio that is the sum of signal-tonoise ratios of the $L$ received signals. Consider the use of MRC for a particular symbol in the symbol sequence and consider the pre-combining samples of $L$ copies of an arbitrary transmitted symbol $d$ at the output of the matched filter. Thus, the $l^{t h}$ sub-channel received signal is given by $y_{l}=h_{l} d+n_{l}, \quad l=1,2, \ldots, L$. In vector form, the received signal is given by $\mathbf{y}=\mathbf{h} d+\mathbf{n}$. The MRC assumes perfect channel estimation. When the received vector is a column vector, the co-phasing/weighting normalized 'matched filter' is given by $\frac{\mathbf{h}^{\dagger}}{\|\mathbf{h}\|}$, where $\dagger$ denotes conjugate transpose of a vector and $\|\cdot\|$ is the norm of vector. Thus, the sufficient statistic for coherent demodulation is $\frac{\mathbf{h}^{\dagger}}{\|\mathbf{h}\|} \mathbf{y}$ and the the MRC decision variable is

$$
X=\frac{\mathbf{h}^{\dagger}}{\|\mathbf{h}\|}(\mathbf{h} d+\mathbf{N})
$$

where $\frac{\mathbf{h}^{\dagger}}{\|\mathbf{h}\|} \mathbf{N}$ has a variance $\sigma^{2}$. The noise-free received symbol is given by

$$
\tilde{X}=\|\mathbf{h}\| d
$$

where energy in the received symbol is clearly $E_{d}=\|\mathbf{h}\| d(\|\mathbf{h}\| d)^{*}=\|\mathbf{h}\|^{2}|d|^{2}$.

Thus, the instantaneous MRC output SNR, given a symbol $d$ is

$$
\mathrm{SNR}_{d}=\frac{\|\mathbf{h}\|^{2}|d|^{2}}{\sigma^{2}}=\sum_{l} \frac{\left|h_{l}\right|^{2}|d|^{2}}{\sigma^{2}}=\sum_{l} \mathrm{SNR}_{l}
$$

where $\mathrm{SNR}_{l}=\frac{\left|h_{l}\right|^{2}|d|^{2}}{\sigma^{2}}$ is the instantaneous SNR of the pre-combining $l_{t h}$ copy of symbol $d$. Thus, MRC achieves the maximum output signal-to-noise ratio, which is the sum of $L$ input SNRs of the multiple copies of the received signal. While non-coherent demodulation is possible, most techniques lead to lower effective SNR which degrades performance. Non-coherent demodulation is beyond the scope of this paper.

\subsection{Orthogonal Space-Time Block Coding}

In a receiver, the channel-tap weighted copies are available at the input of the combiner. Multiple transmit antennas alone cannot provide separate copies of the symbol at the receiver because the single-antenna receiver receives the sum of these copies without the ability to separate them for combining. A combination of transmit antenna diversity and time diversity provides the receiver sufficient statistics to separate the copies for combining, which is achieved by applying OSTBC in the transmitter. We will assume fixed throughput and fixed transmitted power for proper comparison to a non-diversity system. We assume that the transmit antennas have sufficient inter-element spacing in order to have uncorrelated paths. If the $m$ symbols $\check{\mathbf{d}}=\left[\begin{array}{lllll}d_{1} & d_{2} & \cdots & d_{m}\end{array}\right]$ to be transmitted are coded by the OSTBC $\mathbf{G}$, then the received signal is given by

$$
\mathbf{Z}=\mathbf{G h}+N
$$

The complex code matrix $\mathbf{G}$ is an $L_{s}$-by- $L_{t}$, where $L_{t}$ is the number of transmit antennas for $m$ complex symbols transmitted over $L_{s}$ symbol times. Thus, the code rate is $R=m / L_{s} . N$ is the $L_{s}$-by-1 additive white Gaussian noise (AWGN) due to the receiver with covariance matrix $\sigma^{2} \mathbf{I}_{L_{s} X L_{s}}$, and $\mathbf{h}$ is the $L_{t}$-by-1 complex channel attenuation where $\mathbf{h}=\left[\begin{array}{llll}h_{1} & h_{2} & \cdots & h_{L_{t}}\end{array}\right]^{t}$. The OSTBC $\mathbf{G}$ is designed such that the received signal can be transformed to a convenient form

$$
\check{\mathbf{Y}}=\mathbf{H} \check{\mathbf{d}}+\check{\mathbf{N}}
$$

where $\check{\mathbf{d}}$ is the symbol sequence defined previously and the $L_{t}$-by-1 $\check{\mathbf{N}}$ is the preserved noise vector, i.e., the variance is preserved and the covariance of the noise vector is given by $\sigma^{2} \mathbf{I}_{L_{t} X L_{t}}$. The $L_{t}$-by- $m$ matrix $\mathbf{H}$ is the termed as the channel tap matrix whose row entries are mainly derived from $\mathbf{h}$ such that the columns of $\mathbf{H}$ are orthogonal to each other, i.e., $\|\mathbf{h}\|^{2} \mathbf{I}_{m X m}=\mathbf{H}^{\dagger} \mathbf{H}$ or equivalently $\|\mathbf{h}\|^{2} \mathbf{I}_{m X m}=\mathbf{H}^{t} \mathbf{H}^{*}$, where $\|\mathbf{h}\|$ is clearly the norm of $\mathbf{h}$.

\subsection{BER for Rayleigh Fading with Diversity in AWGN}

When OSTBC and MRC are used for MIMO configuration, the diversity order is the $L=L_{t} L_{r}$, where $L_{r}$ is the number of receiver antennas. When the sub-channel tap magnitudes $\left|h_{l}\right|$ are Rayleigh distributed with normalized 
mean square value $\mathrm{E}\left[\left|h_{l}\right|^{2}\right]=1$, the combining channel-tap magnitude squared $\|h\|^{2}=\sum_{l=1}^{L}\left|h_{l}\right|^{2}$ has a chi-square density function with $2 L$ degrees of freedom. The bit error rate (BER) is approximated by [25]

$$
P_{b} \approx \frac{N_{n}}{\log _{2} M}\left(\frac{1-\mu}{2}\right)^{L} \sum_{l=0}^{L-1}\left(\begin{array}{c}
L-1+l \\
l
\end{array}\right)\left(\frac{1+\mu}{2}\right)^{l},
$$

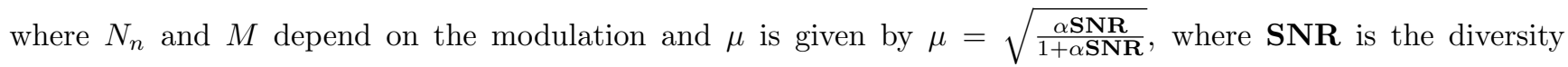
signal-to-noise ratio and $\alpha$ is a modulation-dependent parameter such that $\alpha \mathbf{S N R}=\frac{1}{2}\left(\frac{d_{\min }}{2 \sigma}\right)^{2}$, where $d_{\min }$ is the minimum Euclidean distance of the I-Q signal set and $\sigma$ is the standard deviation (root-variance) of noise.

\section{Complex Spreading vs Traditional Spreading}

A SS modulated signal is a signal that has a much larger bandwidth than its narrowband counterpart via spreading in the frequency domain. The jammer in the channel adds on to the received signal. On receive, the narrowband signal is re-formed via de-spreading. This results in an effective reduction of the magnitude of the power spectral density (PSD) of the jamming signal and thus, the effectiveness of jamming is reduced. The gain, called spreading gain, only applies to the jamming interference and not the receiver noise since the receiver noise is effectively the system noise floor. Assume a bit sequence (-1s and $1 \mathrm{~s})$ with a bit rate of $R_{b}$, where $T_{b}$ is the bit time. Assume a periodic pseudo-noise $(\mathrm{PN})$ sequence of $N_{c}$ chips $(-1 \mathrm{~s}$ and $1 \mathrm{~s})$ with a chip time of $T_{c}$, where $T_{b}=N_{c} T_{c}$. To perform DS spreading, each data bit waveform is multiplied by the PN sequence. The chip rate is clearly $R_{c}=N_{c} R_{b}$ times the bit rate and thus the SS signal bandwidth is $N_{c}$ times the bandwidth of the narrowband signal. The PN sequence period may be selected to be an integer multiple of the bit duration. The number $N_{c}$ is also known as the spread factor of the SS signal. The analog-to-digital conversion (ADC) usually happens at the IF frequency or higher sampling rate for direct-downconversion receivers, virtually ensuring that all receive complex signal processing happens in sampled signal space. For convenience, we may think of our sampling time to be normalized, i.e., $T_{c}=1$.

\subsection{Complex Spreading Procedure}

Let $\mathbf{D}$ be the row of $m$ symbols to be transmitted, where $D_{n}=d_{n}+j \hat{d}_{n}$ be an arbitrary complex symbol from $\mathbf{D}$. The $d$ bit is transmitted via the I channel and $\hat{d}$ bit is transmitted via the $\mathrm{Q}$ channel. Of course, the constellation symbol depends on the chosen modulation. Let a complex spreading function be $\mathbf{c}=\mathbf{c}_{I}+j \mathbf{c}_{Q}$. We can spread all the symbols $\mathbf{D}$ with the same complex code or each symbol $D_{n}$-symbol can be spread with a different code. If we use the same complex code, then the spread signal $\mathbf{S}$ is given by

$$
\mathbf{S}=\frac{\mathbf{c}}{\|\mathbf{c}\|} \mathbf{D}
$$

where the normalization of the code c is utilized to preserve the symbol energies in D. For an arbitrary $D_{n}$, $\mathbf{s}_{n}=D_{n} \frac{\mathbf{c}}{\|\mathbf{c}\|}$, thus the complex spread symbol takes the form $\mathbf{s}_{n}=\mathbf{s}_{n I}+j \mathbf{s}_{n Q}$ where the I and Q spread symbols are given by

$$
\begin{aligned}
\mathbf{s}_{n I} & =\frac{1}{\|\mathbf{c}\|}\left(d_{n} \mathbf{c}_{I}-\hat{d}_{n} \mathbf{c}_{Q}\right) \\
\mathbf{s}_{n Q} & =\frac{1}{\|\mathbf{c}\|}\left(\hat{d}_{n} \mathbf{c}_{I}+d_{n} \mathbf{c}_{Q}\right) .
\end{aligned}
$$

The top block diagram of Figure 1 shows the classical dual-channel procedure while the bottom block diagram illustrates the complex spreading procedure together with OSTBC to accommodate the MIMO technique. In dual-channel spread spectrum, the the I and Q signals are spread by separate codes and there is no provision to perform (8). When (8) is not performed, then cross-talk results.

\subsection{Received Signal in Noise and Jamming}

The jamming signal adds on to the received signal. Moreover, the complex spreading in the transmitter has to be accounted for such that (5) is modified to

$$
\mathbf{Y}=\mathbf{S H}^{t}+\mathbf{N}+\mathbf{J}=\frac{\mathbf{c D} \mathbf{H}^{t}}{\|\mathbf{c}\|}+\mathbf{N}+\mathbf{J}
$$

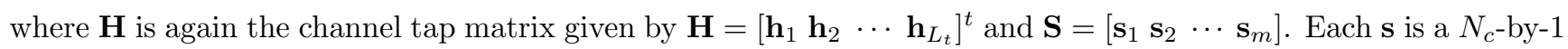
column vector and thus $\mathbf{S}$ is an $N_{c}$-by- $m$ matrix. The received noise measurement $\mathbf{N}=\left[\begin{array}{lllll}\mathbf{n}_{1} & \mathbf{n}_{2} & \cdots & \mathbf{n}_{N_{c}}\end{array}\right]^{t}$, and the jamming interference measurement $\mathbf{J}=\left[\mathbf{j}_{1} \mathbf{j}_{2} \cdots \mathbf{j}_{N_{c}}\right]^{t}$ are $N_{c}$-by- $L_{t}$ matrices. We point out that the interferences we consider in this paper are barrage noise, pulsed noise, and tone jamming. Multi-device and/or self jamming are not considered. 

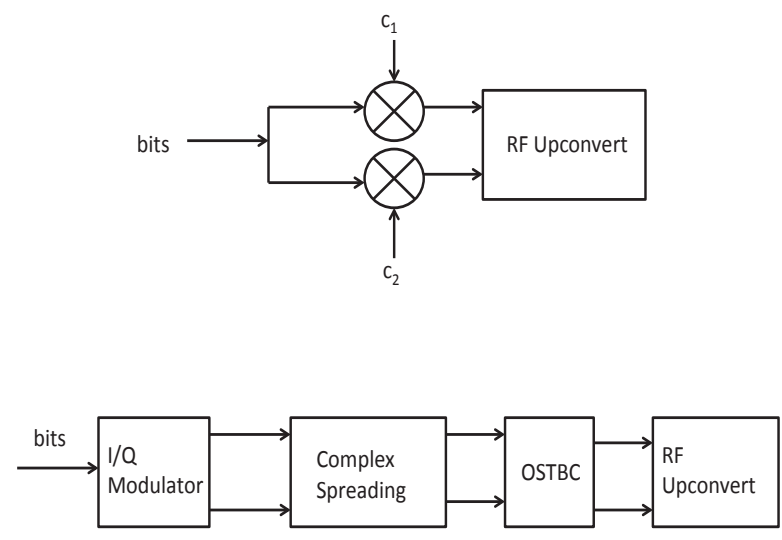

Figure 1. a) Classical dual-channel spreading, b) Complex spreading procedure

\subsection{Complex Despreading Procedure}

The goal of the despreading operation in a receiver is to recover the original complex symbol sequence $\mathbf{D}$ while mitigating the effect of the jamming interference. Here we show that we can recover the transmitted symbols in Rayleigh fading. The complex despreading operator or correlator is given by $\frac{\mathbf{c}^{\dagger}}{\|\mathbf{c}\|}$. Thus, the despread output vector is produced by performing the despreading operation on the whole measurement matrix $\mathbf{Y}$, i.e.,

$$
\mathbf{x}=\frac{\mathbf{c}^{\dagger}}{\|\mathbf{c}\|} \mathbf{Y}=\mathbf{x}_{D}+\mathbf{n}_{D}+\mathbf{j}_{D}
$$

where $\mathbf{n}_{D}=\frac{\mathbf{c}^{\dagger}}{\|\mathbf{c}\|} \mathbf{N}$ is the noise due to the receiver, $\mathbf{j}_{D}=\frac{\mathbf{c}^{\dagger}}{\|\mathbf{c}\|} \mathbf{J}$ is the interference due to a jammer, and

$$
\mathbf{x}_{D}=\frac{\mathbf{c}^{\dagger}}{\|\mathbf{c}\|} \mathbf{S} \mathbf{H}^{t}=\frac{\mathbf{c}^{\dagger}}{\|\mathbf{c}\|^{2}} \mathbf{c D H} \mathbf{H}^{t}=\mathbf{D} \mathbf{H}^{t}
$$

is the received signal without interference. It is clear that the desired symbol sequence $\mathbf{D}$ is available in $\mathbf{x}_{D}$, which is scaled by the channel tap matrix. The use of MRC such that $\mathbf{D}$ is completely retrieved will addressed later.

While (11) is compact, the operations that give intuition to this result is hidden in its simplicity. To best illustrate the despreading operation, it is best to look at one row of vector of $\mathbf{Y}$, i.e., one diversity path. Indeed, if we use an arbitrary spreading code $\mathbf{c}$ for each symbol $D_{n}$, then it would be advantageous for us to look at the output for each path. Recall the receiver measurement $\mathbf{Y}=\left[\begin{array}{llll}\mathbf{y}_{1} & \mathbf{y}_{2} & \cdots & \mathbf{y}_{L_{t}}\end{array}\right]$, where $\mathbf{y}$ clearly corresponds to the signal received from an arbitrary path. Now, consider any arbitrary column vector $\mathbf{y}$ received without noise and interference given by

$$
\mathbf{y}=\mathbf{S h}^{t}=\sum_{n=1}^{m} h_{n} \mathbf{s}_{n} .
$$

If we let the interference-free despread measurement (11) be $\mathbf{x}_{D}=\left[\begin{array}{ll}x_{D 1} & x_{D 2} \\ \cdots & x_{D L_{t}}\end{array}\right]$, then the corresponding $x_{D}$ for an arbitrary $\mathbf{y}$ in (12) is

$$
x_{D}=\frac{\mathbf{c}^{\dagger}}{\|\mathbf{c}\|} \mathbf{y}=\frac{1}{\|\mathbf{c}\|} \sum_{n=1}^{m} h_{n} \mathbf{c}^{\dagger} \mathbf{s}_{n}
$$

Recalling (8), then (13) takes the form

$$
x_{D}=\frac{1}{\|\mathbf{c}\|} \sum_{n=1}^{m} h_{n}\left\{\left(\mathbf{c}_{\mathbf{I}}^{\dagger} \mathbf{s}_{n I}+\mathbf{c}_{\mathbf{Q}}^{\dagger} \mathbf{s}_{n Q}\right)+j\left(\mathbf{c}_{\mathbf{I}}^{\dagger} \mathbf{s}_{n Q}-\mathbf{c}_{\mathbf{Q}}{ }^{\dagger} \mathbf{s}_{n I}\right)\right\}
$$

It can be shown that

$$
\begin{aligned}
& \mathbf{c}_{\mathbf{I}}^{\dagger} \mathbf{s}_{n I}+\mathbf{c}_{\mathbf{Q}^{\dagger} \mathbf{s}_{n Q}}=\frac{d_{n}}{\|\mathbf{c}\|}\left(\mathbf{c}_{\mathbf{I}}^{\dagger} \mathbf{c}_{\mathbf{I}}+\mathbf{c}_{\mathbf{Q}}^{\dagger} \mathbf{c}_{\mathbf{Q}}\right)=d_{n}\|\mathbf{c}\| \\
& \mathbf{c}_{\mathbf{I}}^{\dagger} \mathbf{s}_{n Q}-\mathbf{c}_{\mathbf{Q}}^{\dagger} \mathbf{s}_{n I}=\frac{\hat{d}_{n}}{\|\mathbf{c}\|}\left(\mathbf{c}_{\mathbf{I}}^{\dagger} \mathbf{c}_{\mathbf{I}}+\mathbf{c}_{\mathbf{Q}}^{\dagger} \mathbf{c}_{\mathbf{Q}}\right)=\hat{d}_{n}\|\mathbf{c}\| .
\end{aligned}
$$

Applying (15) to (14) results in

$$
x_{D}=\sum_{n=1}^{m} h_{n}\left(d_{n}+j \hat{d}_{n}\right)=\sum_{n=1}^{m} h_{n} D_{n}=\mathbf{h} D_{n} .
$$

Thus, we can form a despread vector by stacking all the $L$ channel-attenuated symbols (16) of the transmitted sequence. Indeed, this yields the desired result, i.e., the despread row vector (11). If we choose to have a column vector, then we can easily take the transpose which is $\mathbf{x}_{D}^{t}=\mathbf{H} \mathbf{D}^{t}$. 


\subsection{Detection via MRC}

Finally, it is our interest to detect the symbol sequence $\mathbf{D}$ via MRC, which assumes the channel matrix to be known via estimation. For a received signal in column vector, the MRC operation takes the form $\frac{\mathbf{H}^{\dagger}}{\|\mathbf{h}\|} \mathbf{x}^{t}$ and for row vector, it takes the form $\mathbf{x} \frac{\mathbf{H}^{*}}{\|\mathbf{h}\|}$. We can choose either method. Utilizing the latter method, the final received symbol sequence in noise and jamming interference is given by

$$
\mathbf{w}=\mathbf{x} \frac{\mathbf{H}^{*}}{\|\mathbf{h}\|}=\mathbf{z}+\mathbf{n}_{R}+\mathbf{j}_{R}
$$

where $\mathbf{z}=\mathbf{x}_{D} \frac{\mathbf{H}^{*}}{\|\mathbf{h}\|}, \mathbf{n}_{R}=\mathbf{n}_{D} \frac{\mathbf{H}^{*}}{\|\mathbf{h}\|}$, and $\mathbf{j}_{R}=\mathbf{j}_{D} \frac{\mathbf{H}^{*}}{\|\mathbf{h}\|}$. The dimension of $\mathbf{w}$ at the output the MRC operation is 1 -by- $m$, which matches the symbol sequence sent. Without any interference, $\mathbf{w}$ is simply

$$
\begin{aligned}
\mathbf{z} & =\mathbf{x}_{D} \frac{\mathbf{H}^{*}}{\|\mathbf{h}\|}=\mathbf{D H}^{t} \frac{\mathbf{H}^{*}}{\|\mathbf{h}\|} \\
& =\mathbf{D}\|\mathbf{h}\| \mathbf{I}_{m X m}=\|\mathbf{h}\| \mathbf{D},
\end{aligned}
$$

where $\mathbf{D}$, albeit scaled by $\|\mathbf{h}\|$ is available for detection. Notice that (18) agrees with (2) when an arbitrary symbol is considered. Indeed the total energy in the whole sequence is easily calculated to be $\|\mathbf{h}\|^{2}\|\mathbf{D}\|^{2}$, where the energy contribution for an arbitrary symbol is $\|\mathbf{h}\|^{2}\left\|D_{n}\right\|^{2}$, which also agrees with the energy for (2). In other words, the use of MRC for the Rayleigh fading channel together with proper complex spreading and despreading operation does not result in loss of signal energy. We are left however with calculating the jammer contribution due to interference which is the subject of the next section.

For detection of arbitrary symbol $D_{n}=d_{n}+j \hat{d}_{n}$, we utilize the minimum Euclidean distance criterion which would allow us to use (6) but to be modified such that the jamming interference is accounted for.

\section{Interference Power Calculations: Covariances}

The received measurement goes through two major signal processing operations: despreading and MRC operations. To find the noise power of the sufficient vector statistic out of the MRC, it is necessary to analyze the noise and jammer transformations after both the despreading operation and MRC. Recall that after despreading, the received measurement is (10). We need to find the covariances of $\mathbf{n}_{D}=\frac{\mathbf{c}^{\dagger}}{\|\mathbf{c}\|} \mathbf{N}$ and $\mathbf{j}_{D}=\frac{\mathbf{c}^{\dagger}}{\|\mathbf{c}\|} \mathbf{J}$, where $\mathbf{J}$ is due to any of the three jamming interferences: barrage noise, pulsed noise, and tone jamming. The received measurement post-MRC is (17), where we need to find the covariances of $\mathbf{n}_{R}=\mathbf{n}_{D} \frac{\mathbf{H}^{*}}{\|\mathbf{h}\|}$, and $\mathbf{j}_{R}=\mathbf{j}_{D} \frac{\mathbf{H}^{*}}{\|\mathbf{h}\|}$.

\section{$4.1 \quad$ Receiver Noise}

Recalling that $\mathbf{N}=\left[\begin{array}{llll}\mathbf{n}_{1} & \mathbf{n}_{2} & \cdots & \mathbf{n}_{N_{c}}\end{array}\right]^{t}$, where an arbitrary row vector $\mathbf{n}$ is a 1 -by- $L_{t}$. The noise is the simply the AWGN receiver noise, i.e., $\mathbf{n} \sim \mathcal{C N}\left(\mathbf{0}, \sigma_{n}^{2} \mathbf{I}_{L_{t} X L_{t}}\right)$. However, our interest is finding the covariance of the noise vector after despreading. Let $\mathbf{n}_{D}=\frac{\mathbf{c}^{\dagger}}{\|\mathbf{c}\|} \mathbf{N}=\left[\begin{array}{llll}\eta_{1} & \eta_{2} & \cdots & \eta_{L_{t}}\end{array}\right]$, then its covariance is given by

$$
\operatorname{cov}\left(\mathbf{n}_{D}\right)=\mathrm{E}\left[\mathbf{n}_{D}^{\dagger} \mathbf{n}_{D}\right]=\mathrm{E}\left[\left(\frac{\mathbf{c}^{\dagger}}{\|\mathbf{c}\|} \mathbf{N}\right)^{\dagger} \frac{\mathbf{c}^{\dagger}}{\|\mathbf{c}\|} \mathbf{N}\right]
$$

Although it takes very lengthy derivation, it can be shown that (19) reduces to

$$
\operatorname{cov}\left(\mathbf{n}_{D}\right)=\sigma_{n}^{2} \mathbf{I}_{L_{t} X L_{t}}
$$

which says that the noise samples are still uncorrelated. The proof and derivation from (19) to (20) is shown in the Appendix.

The arbitrary noise sample $\eta$ is given by

$$
\eta=\frac{\mathbf{c}^{\dagger}}{\|\mathbf{c}\|} \mathbf{n}
$$

where $\mathbf{n}$ is row vector corresponding to an arbitrary path in the noise measurement $\mathbf{N}$. It can be shown that variance of $(21)$ is $\operatorname{var}(\eta)=\sigma_{n}^{2}$ which corresponds to the result $(20)$.

Detection eventually happens after the MRC, i.e., the MRC noise vector is given by $\mathbf{n}_{R}=\mathbf{n}_{D} \frac{\mathbf{H}^{*}}{\|\mathbf{h}\|}=\left[n_{R_{1}} n_{R_{2}} \cdots n_{R_{L_{t}}}\right]$. The covariance of the MRC noise is given by

$$
\begin{aligned}
\operatorname{cov}\left(\mathbf{n}_{R}\right) & =\mathrm{E}\left[\left(\mathbf{n}_{D} \frac{\mathbf{H}^{*}}{\|\mathbf{h}\|}\right)^{\dagger} \mathbf{n}_{D} \frac{\mathbf{H}^{*}}{\|\mathbf{h}\|}\right] \\
& =\sigma_{n}^{2} \frac{\mathbf{H}^{t}}{\|\mathbf{h}\|} \mathbf{I}_{L_{t} X L_{t}} \frac{\mathbf{H}^{*}}{\|\mathbf{h}\|}=\sigma_{n}^{2} \mathbf{I}_{m X m}
\end{aligned}
$$

which says the noise samples are still uncorrelated. It can also be shown that the variance of an arbitrary noise sample $n_{R}$ is $\sigma_{n}^{2}$. 


\subsection{Barrage Noise Jamming}

Here we consider that jamming is due to barrage noise, which is modeled as broadband noise, i.e., AWGN. For the unspread system, let the jamming sample to have $\sim \mathcal{C N}\left(0, \sigma_{B}^{2}\right)$. For the I and $\mathrm{Q}$ channel noise sample in a spread spectrum system, let the variance of the complex noise sample $\sigma_{B^{\prime}}^{2}$. It is well known that for the spread system the power of the noise is decreased by the spreading gain $N_{c}$ [17-18], [20-23]. Thus, the complex jamming noise sample is distributed according to $\sim \mathcal{C N}\left(0, \sigma_{B^{\prime}}^{2}=\frac{\sigma_{B}^{2}}{N_{c}}\right)$.

The despread jamming vector $\mathbf{j}_{D}=\frac{\mathbf{c}^{\dagger}}{\|\mathbf{c}\|} \mathbf{J}=\left[\begin{array}{llll}\varphi_{1} & \varphi_{2} & \cdots & \varphi_{L_{t}}\end{array}\right]$ and its covariance is

$$
\operatorname{cov}\left(\mathbf{j}_{D}\right)=\mathrm{E}\left[\left(\mathbf{j}_{D}\right)^{\dagger} \mathbf{j}_{D}\right]=\mathrm{E}\left[\left(\frac{\mathbf{c}^{\dagger}}{\|\mathbf{c}\|} \mathbf{J}\right)^{\dagger} \frac{\mathbf{c}^{\dagger}}{\|\mathbf{c}\|} \mathbf{J}\right] .
$$

Following the same derivation technique from Appendix A and accounting for the spreading gain, (23) reduces to

$$
\operatorname{cov}\left(\mathbf{j}_{D}\right)=\frac{\sigma_{B}^{2}}{N_{c}} \mathbf{I}_{L_{t} X L_{t}}
$$

which says that the jamming interference samples at the output of the despreader are still uncorrelated. The arbitrary despread jamming noise sample $\varphi$ given an arbitrary path is given by

$$
\varphi=\frac{\mathbf{c}^{\dagger}}{\|\mathbf{c}\|} \mathbf{j}
$$

where $\operatorname{var}(\varphi)=\frac{\sigma_{B}^{2}}{N_{c}}$.

The MRC barrage jamming noise vector is given by $\mathbf{j}_{R}=\mathbf{j}_{D} \frac{\mathbf{H}^{*}}{\|\mathbf{h}\|}=\left[j_{R_{1}} j_{R_{2}} \cdots j_{R_{L_{t}}}\right]$. Following the straightforward derivation in (22), its covariance is

$$
\operatorname{cov}\left(\mathbf{j}_{R}\right)=\mathrm{E}\left[\left(\mathbf{j}_{D} \frac{\mathbf{H}^{*}}{\|\mathbf{h}\|}\right)^{\dagger} \mathbf{j}_{D} \frac{\mathbf{H}^{*}}{\|\mathbf{h}\|}\right]=\frac{\sigma_{B}^{2}}{N_{c}} \mathbf{I}_{m X m}
$$

which says the barrage noise samples are uncorrelated. It follows that the variance of an arbitrary barrage noise sample $j_{R}$ is $\frac{\sigma_{B}^{2}}{N_{c}}$.

\subsection{Pulsed Noise Jamming}

Here we consider that jamming is due to pulsed noise jamming, which is broadband jamming noise received in periodic spurt. The duty cycle is given by $\rho$, the percentage of time the that the jammer is on, i.e., $0<\rho \leq 1$. The jammer is considered to be turned off when $\rho=0$. It should be clear that the barrage noise jamming is just a special case of the pulsed noise jamming where $\rho=1$. For $\rho<1$, to preserve the same power as barrage jamming, the power is scaled by $\rho$. For the unspread system, let the jamming sample be distributed according to $\sim \mathcal{C N}\left(0, \frac{\sigma_{P}^{2}}{\rho}\right)$ where $\sigma_{P}^{2}$ is the variance of the pulsed noise interference. For the spread system, let the variance of the corresponding complex interference sample be $\sigma_{P^{\prime}}^{2}$. Accounting for the spreading gain and duty cycle, the interference sample is distributed according to $\sim \mathcal{C N}\left(0, \frac{\sigma_{P}^{2}}{\rho N_{c}}\right)$.

The despread jamming vector due to pulsed noise jamming is $\mathbf{j}_{P}=\frac{\mathbf{c}^{\dagger}}{\|\mathbf{c}\|} \mathbf{J}=\left[\begin{array}{llll}\psi_{1} & \psi_{2} & \cdots & \psi_{L_{t}}\end{array}\right]$. Following the derivation in the Appendix A, the covariance is shown to be

$$
\operatorname{cov}\left(\mathbf{j}_{P}\right)=\frac{\sigma_{P}^{2}}{\rho N_{c}} \mathbf{I}_{L_{t} X L_{t}} .
$$

The arbitrary despread jamming noise sample $\psi$ (corresponding to an arbitrary path) is given by $\varphi=\frac{\mathbf{c}^{\dagger}}{\|\mathbf{c}\|} \mathbf{j}$, where $\operatorname{var}(\psi)=\frac{\sigma_{P}^{2}}{\rho N_{c}}$ is now an expected result.

The MRC pulsed noise vector is given by $\mathbf{j}_{S}=\mathbf{j}_{P} \frac{\mathbf{H}^{*}}{\|\mathbf{h}\|}=\left[j_{S_{1}} j_{S_{2}} \cdots j_{S_{L_{t}}}\right]$. Again, following the straightforward derivation in (22), its covariance is

$$
\operatorname{cov}\left(\mathbf{j}_{S}\right)=\frac{\sigma_{P}^{2}}{\rho N_{c}} \mathbf{I}_{m X m}
$$

The variance of an arbitrary pulsed noise jamming sample $j_{S}$ is $\frac{\sigma_{P}^{2}}{\rho N_{c}}$. 


\subsection{Tone Jamming}

Another common jammer is the tone jammer. In tone jamming, an interference tone at the carrier frequency is sent to jam the carrier frequency. After passband downconversion to baseband, the interference results in a phase offset. We assume the received phase offset on the initial chip to be random from $(-\pi, \pi)$. Since the interference is a sinewave, the phase offset is totally correlated from chip to chip. Recall from (10) that the interference prior to despreading is $\mathbf{J}=\left[\begin{array}{llll}\mathbf{j}_{1} & \mathbf{j}_{2} & \cdots & \mathbf{j}_{N_{c}}\end{array}\right]^{t}$, where an arbitrary $\mathbf{j}$ is given by $\mathbf{j}=\left[\begin{array}{llll}\phi_{1} & \phi_{2} & \cdots & \phi_{L_{t}}\end{array}\right]=\frac{A_{J}}{2}\left[\begin{array}{lll}e^{-j \theta_{1}} & e^{-j \theta_{2}} \cdots e^{-j \theta_{L_{t}}}\end{array}\right]$, where $A_{J}$ is the amplitude of the carrier tone. Due to complete phase correlation, $\mathbf{j}=\frac{A_{J}}{2}\left[e^{-j \theta} e^{-j \theta} \ldots e^{-j \theta}\right]$ and it is clear that the noise covariance $\operatorname{cov}(\mathbf{j})=\mathrm{E}\left[(\mathbf{j})^{\dagger} \mathbf{j}\right]=\left(\frac{A_{J}}{2}\right)^{2} \mathbf{1}_{L_{t} X L_{t}}$ where $\mathbf{1}$ is a matrix with all 1 entries.

The despread noise vector is given by $\mathbf{j}_{Q}=\frac{\mathbf{c}^{\dagger}}{\|\mathbf{c}\|} \mathbf{J}$ where $\mathbf{J}$ is given by

$$
\mathbf{J}=\frac{A_{J}}{2}\left(\begin{array}{cccc}
e^{-j \theta_{11}} & e^{-j \theta_{21}} & \cdots & e^{-j \theta_{L_{t} 1}} \\
e^{-j \theta_{12}} & e^{-j \theta_{22}} & \cdots & e^{-j \theta_{L_{t} 2}} \\
\vdots & \vdots & \ddots & \vdots \\
e^{-j \theta_{1 N_{c}}} & e^{-j \theta_{2 N_{c}}} & \cdots & e^{-j \theta_{L_{t} N_{c}}}
\end{array}\right)
$$

and

$$
\mathbf{j}_{Q}=\frac{A_{J}}{2\|\mathbf{c}\|}\left(\begin{array}{c}
c_{1}^{*} e^{-j \theta_{11}}+c_{2}^{*} e^{-j \theta_{12}}+\cdots+c_{N_{c}}^{*} e^{-j \theta_{1 N_{c}}} \\
c_{1}^{*} e^{-j \theta_{21}}+c_{2}^{*} e^{-j \theta_{22}}+\cdots+c_{N_{c}}^{*} e^{-j \theta_{2 N_{c}}} \\
\vdots \\
c_{1}^{*} e^{-j \theta_{L_{t} 1}}+c_{2}^{*} e^{-j \theta_{L_{t} 2}}+\cdots+e^{-j \theta_{L_{t} N_{c}}}
\end{array}\right)^{t}
$$

This simplifies to (see Appendix B)

$$
\mathbf{j}_{Q}=\frac{A_{J}}{2\|\mathbf{c}\|}\left(\begin{array}{c}
e^{-j \theta_{1}}-j e^{-j \theta_{1}} \\
e^{-j \theta_{2}}-j e^{-j \theta_{2}} \\
\vdots \\
e^{-j \theta_{L_{t}}}-j e^{-j \theta_{L_{t}}}
\end{array}\right)^{t}
$$

The covariance is calculated to be

$$
\operatorname{cov}\left(\mathbf{j}_{Q}\right)=\frac{A_{J}^{2}}{4 N_{c}} \mathbf{I}_{L_{t} X L_{t}}
$$

It follows that the one-sample tone interference $\phi$ has a variance given by $\frac{A_{J}^{2}}{4 N_{c}}$.

The MRC tone interference vector is given by $\mathbf{j}_{T}=\mathbf{j}_{Q} \frac{\mathbf{H}^{*}}{\|\mathbf{h}\|}=\left[\begin{array}{lll}j_{Q_{1}} & j_{Q_{2}} \cdots & j_{Q_{L_{t}}}\end{array}\right]$. Thus, its covariance is shown to be

$$
\operatorname{cov}\left(\mathbf{j}_{T}\right)=\frac{A_{J}^{2}}{4 N_{c}} \mathbf{I}_{m X m}
$$

where the variance of an arbitrary tone interference sample $j_{Q}$ is $\frac{A_{J}^{2}}{4 N_{c}}$.

\section{SINR and BER Results: 16QAM Example}

The general results derived in the previous sections will now be used for a specific modulation to facilitate an example. We evaluate the BER performances of a 16QAM complex-spreading MIMO systems in Rayleigh fading with broadband noise, pulsed noise, and tone jamming. MRC is assumed in the receiver. Since there are $L_{t}$ and $L_{r}$ antennas, the diversity gain is $L=L_{t} L_{r}$. To maintain proper comparison to a single antenna system, symbol energy is adjusted depending on the number of transmit antennas and the rate of the space-time code. The multiplicative scaling is given by $R / L_{t}$. For $L_{t}=2$, we use the Alamouti code which has a code rate $R=1$ and as such, the symbol energy is multiplied by $1 / 2$. For $L_{t}=3$, we use an OSTBC with code rate $R=3 / 4$. As such, the multiplicative scale for the symbol energy is $1 / 4$. For $L_{t}=4$, we the use an OSTBC with code rate $R=3 / 4$. As such, the multiplicative scale for the symbol energy is $3 / 16$. Below are are the calculations of SINR and $\mu$ in (6) to be used in calculating BER. Example results ensue.

\subsection{Barrage Noise}

In a MIMO system, we let be the diversity energy given by $\varepsilon_{s}=E_{s} / L_{t}$, where the average symbol energy $E_{s}=$ $E_{b} \log _{2} M$ and $E_{b}$ is the bit energy. In 16 QAM, there are $M=16$ symbols, $N_{n}=3, \log _{2} M=4, d_{\text {min }}=\sqrt{6 \varepsilon_{s} / 15}$. When evaluating BER in communication systems, it conventional to use $E_{b}$ for bit energy, i.e., $E_{b}=\frac{E s}{\log _{2} M}$. Moreover, it is conventional to represent the interference variances in terms of spectral density, e.g., noise variance in terms of $\sigma_{N}^{2}=\frac{N_{0}}{2}$ and jamming variance in terms of $\sigma_{J}^{2}=\frac{J_{0}^{\prime}}{2}$. When OSTBC and MRC are used for MIMO configuration, the diversity order is the $L=L_{t} L_{r}$. 
Since we both have receiver noise and jamming, the modulation-factor scaled SJNR is now given by

$$
\begin{aligned}
\alpha \mathrm{SJNR} & =\frac{1}{2}\left(\frac{d_{\text {min }}}{2 \sigma_{T}}\right)^{2}=\frac{1}{2} \cdot \frac{6 \varepsilon_{s}}{15 \cdot 4 \frac{\left(N_{0}+J_{0}^{\prime}\right)}{2}} \\
& =\frac{2}{5 L_{t}\left[\left(\frac{E_{b}}{N_{0}}\right)^{-1}+\left(\frac{E_{b}}{J_{0}^{\prime}}\right)^{-1}\right]} .
\end{aligned}
$$

In a non-spread system, we let $\sigma_{B}^{2}=\frac{J_{0}}{2}$. In the despread system, then sample noise variance is $\frac{\sigma_{B}^{2}}{N_{c}}=\frac{J_{0}}{2 N_{c}}$, where clearly $J_{0}^{\prime}=\frac{J_{0}}{N_{c}}$. Thus, $\mu$ in (6) to be used for the BER expression is given by

$$
\mu=\sqrt{\frac{1}{\frac{1}{\alpha S J N R}+1}}=\sqrt{\frac{1}{\frac{5}{2} L_{t}\left[\left(\frac{E_{b}}{N_{0}}\right)^{-1}+\left(\frac{E_{b}}{J_{0}}\right)^{-1} \frac{1}{N_{c}}\right]+1}} .
$$
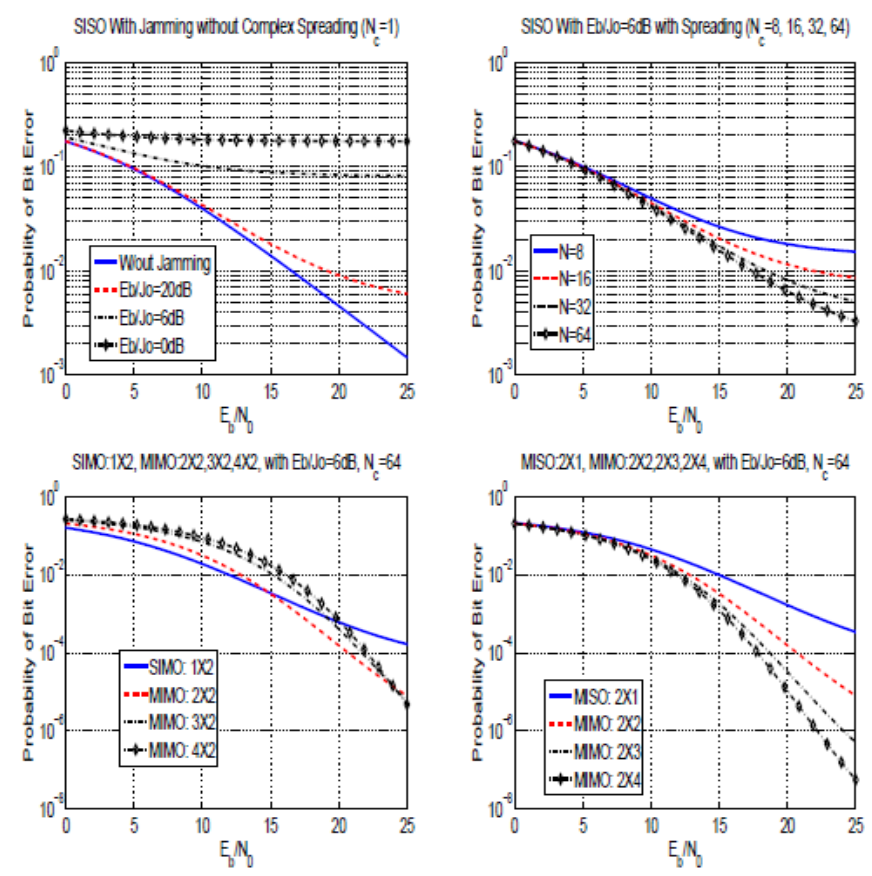

Figure 2. 16-QAM with Broadband Jamming. a) SISO with Jamming, b) SISO with Spreading, c) MIMO: $1 \times 2$, 2x2, 3x2, 4x2, d) MIMO: $2 \times 1,2 \times 2,2 \times 3,2 \times 4$

In Figure 2, the BER performance set for spread 16QAM with broadband jamming in Rayleigh fading is shown. In Figure 2a, the BER curves (versus $E_{b} / N_{0}$ ) as function of decreasing $E_{b} / J_{0}$ are shown for a SISO sytem without spreading. Clearly, the BER worsens as jammer power is increased. The BER for $E_{b} / J_{0}=0 \mathrm{~dB}$ is so degraded that it nearly flattens for $E_{b} / N_{0}>10 \mathrm{~dB}$. In Figure $2 \mathrm{~b}$, the BER curves are shown for systems that use complex spreading. The BER curves are shown for $E_{b} / J_{0}=6 \mathrm{~dB}$ as a function of increasing $N_{c}$. We note the improvement of BER as $N$ is increased. While the improvement is good, note that at $N=64$, the BER is about $3 \times 10^{-3}$, which may be too high for practical systems. Clearly, a combination of spreading and diversity is needed. In Figure 2c, the BER curves are shown as a function of increasing number of transmit antennas. We will not show the combination of 1 transmit antenna and 1 receive antenna (SISO) since this is the case of non-diversity. Here, we use $L_{r}=2$ number of receive antennas since it is known that an amount of diversity in the receiver generally improves BER. For these curves, we will keep $N=64$ and $E_{b} / J_{0}=6 \mathrm{~dB}$ for continued comparison. Here we note that the increase from 1 to 2 transmit antennas greatly improves BER for $E b / N_{0}>15 \mathrm{~dB}$ and minimal degradation for $E b / N_{0}<15$ $\mathrm{dB}$. However, while the increase to 3 and 4 antennas improves BER at large SNRs $\left(E b / N_{0}>25 \mathrm{~dB}\right)$, the BERs are not necessarily better compared to $L_{t}=2$ for low and medium-range SNRs. In fact at $E b / N_{0}<25 \mathrm{~dB}$, the BER suffers considerable degradation. As such, it is clear $L_{t}=2$ is a good choice considering both BER performance and number of transmit antennas. As such we will use $L_{t}=2$ as we increase the number of receive antennas for $N=64$ and $E_{b} / J_{0}=6 \mathrm{~dB}$ for our next set of results. In Figure 2d, BER curves as a function of increasing receive antennas $\left(L_{r}=1,2,3,4\right)$ are shown. Here, it is clear that the BER improves as $L_{r}$ is increased and that there is no penalty when an increase in receive antennas is incurred (except of course receiver complexity). 


\subsection{Pulsed Noise Jamming}

Pulsed noise jamming uses broadband noise but only a percent of the time given by $\rho$, i.e., $\rho$ is the duty cycle of the jammer. For $\rho$ values between 0 and 1, the system performs under receiver noise and jamming signal, For the special case of $\rho=0$, we specify the jammer to be turned off and thus, the receiver interference is just due to the receiver noise. By total probability theorem, the BER is given by

$$
P_{b}=\rho P_{b_{J+N}}+(1-\rho) P_{b_{N}},
$$

where $P_{b_{J+N}}$ is the BER under jamming and receiver noise and $P_{b_{N}}$ is the BER under AWGN only. We recall, for the un-spread system, the PSD of the jamming signal is $J_{0} / 2$. And thus, the corresponding despread PSD is $J_{0}^{\prime}=\frac{J_{0}}{\rho N_{c}}$. If we consider the case where the jamming signal is considerably greater than the receiver noise, i.e., $\left(\frac{E_{b}}{N_{0}}\right)^{-1} \ll\left(\frac{E_{b}}{J_{0}^{\prime \prime}}\right)^{-1}$, then the BER is dominated by the jamming signal, where BER becomes $P_{b} \approx \rho P_{b_{J}}$ and SIR becomes SJR.

For 16-QAM, the parameter $\mu$ simplifies to

$$
\mu=\sqrt{\frac{1}{\frac{1}{\alpha S J R}+1}}=\sqrt{\frac{1}{\frac{5 L_{t}}{2 N \rho}\left(\frac{E_{b}}{J_{0}}\right)^{-1}+1}} .
$$

In Figure 3, the BER performance sets for 16-QAM with pulsed noise jamming are shown. In Figure 3a, the BER curves (versus $E b / J_{0}$ ) as function of increasing $\rho$ are shown for a SISO sytem without spreading. As expected, the BER worsens as $\rho$ is increased. Notice, however that the BER curves converge for large SJR which says that $\rho$ is not a factor as long as the SJR is considerably large. In Figure 3b, the BER curves are shown for $\rho=0.1$ as a function of increasing $N$. As expected, BER improves as $N$ is increased. In Figure 3c, the BER curves are shown for a 2x2 MIMO system with $N=64$ as a function of increasing $\rho$. Here, we note all the BERs improve with the $2 \times 2$ diversity gain. However, the improvement is less effective as $\rho$ decreases. For example, we note the effectiveness of $\rho=0.01$ as a jammer value, i.e., the BER corresponding to it remains farily flat for $E_{b} / J_{0}<5$ $\mathrm{dB}$. That is, if a system is in Rayleigh fading and is known to employ a MIMO technique, the jammer is better off using a practical small $\rho$ (from the jammer's perspective), which is counter to what is observed in Figure 3a, where it was noticed that the higher the duty cycle, the more effective the jammer would be. In Figure $3 \mathrm{~d}$, the BER curves are shown for $2 \times 4$ MIMO system. All BERs improve with the $2 \times 4$ diversity gain and we again note the effectiveness $\rho=0.01$ as a jammer value.
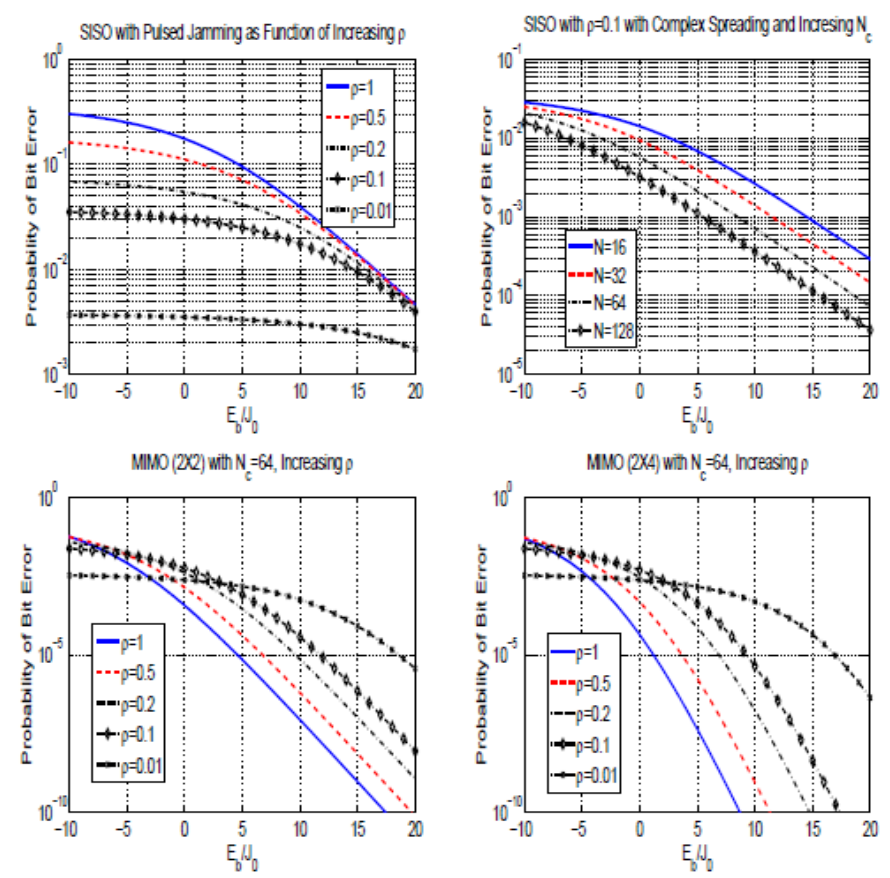

Figure 3. 16-QAM with Pulsed Jamming. a) SISO with Jamming and increasing $\rho$, b) SISO with Spreading, c) MIMO (2x2) and increasing $\rho$, d) MIMO (2x4) and increasing $\rho$ 


\subsection{Tone Jamming}

The modulation-scaled SJNR is given by $\alpha \mathrm{SJNR}=\frac{1}{2} \frac{d_{\min }^{2}}{4 \sigma_{T}^{2}}$. Recall, in 16-QAM, there are $M=16$ symbols, $N_{n}=3, \log _{2} M=4, d_{\min }=\sqrt{6 \varepsilon_{s} / 15}$, and $\varepsilon_{s}=E_{s} / L_{t}$. Thus,

$$
\alpha \mathrm{SJNR}=\frac{1}{2} \frac{6 \varepsilon_{s} / 15}{4\left(\frac{N_{0}}{2}+\frac{A_{J}^{2}}{4 N_{c}}\right)}=\frac{E_{s}}{20 L_{t}\left(\frac{N_{0}}{2}+\frac{A_{J}^{2}}{4 N_{c}}\right)} .
$$

We recall that the bit energy is $E_{b}=\frac{A^{2} N_{c}}{2}$, where $A$ is the carrier amplitude and thus

$$
\alpha \mathrm{SJNR}=\frac{2}{5 L\left[\left(\frac{E_{b}}{N_{0}}\right)^{-1}+\left(\frac{A^{2}}{A_{J}^{2}}\right)^{-1} \frac{1}{N_{c}^{2}}\right]} .
$$

For 16QAM, it can be shown that $\mu$, now a function of both the tone jamming and receiver noise, is given by

$$
\mu=\sqrt{\frac{1}{\frac{1}{\alpha S J N R}+1}}=\sqrt{\frac{1}{\frac{5 L_{t}}{2}\left[\left(\frac{E_{b}}{N_{0}}\right)^{-1}+\left(\frac{A^{2}}{A_{J}^{2}}\right)^{-1} \frac{1}{N_{c}^{2}}\right]+1}} .
$$

In Figure 4, the BER performance set for DS-QPSK with tone jamming is shown. In Figure 4a, the BER curves
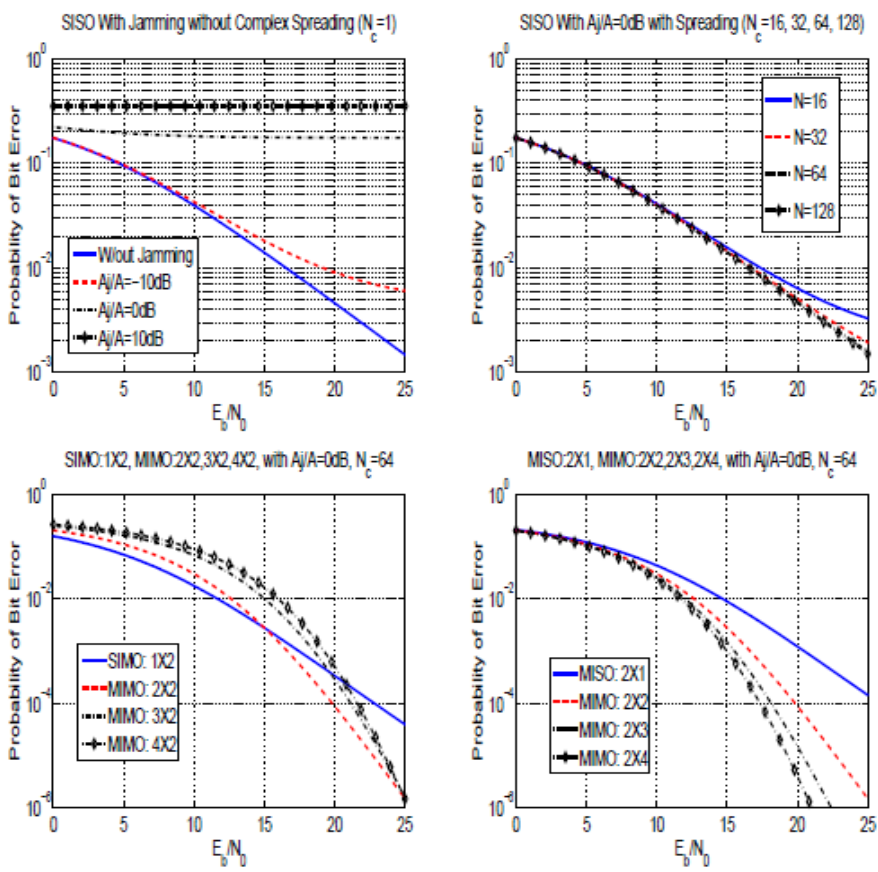

Figure 4. 16-QAM with Tone Jamming. a) SISO with Jamming, b) SISO with Spreading, c) MIMO: $1 \times 2$, 2x2, 3x2, 4x2, d) MIMO: $2 \times 1,2 \times 2,2 \times 3,2 \times 4$

(versus $E_{b} / N_{0}$ ) as function of increasing $A_{J} / A$ are shown for a SISO sytem without spreading. As expected, the BER worsens as jammer power is increased. The BERs for $A_{J} / A=0 \mathrm{~dB}$ becomes almost constant at $E_{b} / N_{0}>5 \mathrm{~dB}$. At $A_{J} / A=10 \mathrm{~dB}$, the BER is so degraded that it is almost constant for the $E_{b} / N_{0}$ range presented. In Figure $4 \mathrm{~b}$, the BER curves are shown for $A_{J} / A=0 \mathrm{~dB}$ as a function of increasing $N$. As expected, BER improves as $N$ is increased. Notice the dramatic BER improvement for $N=16$. Yet, there is little improvement for increasing $N$, which suggests that both spreading and diversity may be needed for practical BER improvement. In Figure 4c, the BER curves are shown for systems as a function of increasing number of transmit antennas. Here, we use $L_{r}=2$ number of receive antennas. Here we note that the increase from 1 to 2 transmit antennas improves BER for $E_{b} / N_{0}>15 \mathrm{~dB}$ and minimal degradation for $E_{b} / N_{0}<15 \mathrm{~dB}$. We note the increase to 3 and 4 antennas does not improve BER compared to 1 transmit antenna until large very SNRs $\left(E_{b} / N_{0}>20 \mathrm{~dB}\right)$. Indeed, compared to 2 transmit antennas, the BER improvement (for 3 and 4 transmit-antenna systems) does not occur until $E_{b} / N_{0}>25$ dB. Moreover, the degradation in BER compared to the 1 transmit-antenna system for $E_{b} / N_{0}<20 \mathrm{~dB}$ is relatively significant. Thus, the use of two transmit antennas is warranted. As such, we will use $L_{t}=2$ as we increase the number of receive antennas for $N=64$ and $A_{J} / A=0 \mathrm{~dB}$ for the next set of BER curves. In Figure $4 \mathrm{~d}$, BER curves as a function of increasing receive antennas $\left(L_{r}=1,2,3,4\right)$ are shown. BER improves as $L_{r}$ is increased. 


\section{Summary and Conclusion}

Some systems may experience fading and jamming at the same time. Thus, there is a need to characterize error performance when both are present. It has been shown that the performance degradation is quite severe when both are present. While it is intuitive to use both MIMO and SS techniques to mitigate the problem, there is a need to characterize BER performances are as result. In this work, we analyzed the effects of both interferences and the derive performance expressions with the use of MIMO and SS. We use a 16QAM as an example modulation to evaluate performance improvements. While performance improvements due to MIMO and SS combined are mostly significant for all three jamming interferences in Rayleigh fading, the effect of pulse jamming turns out to be interesting. For some duty cycle values, the performance improvement is not as significant compared to other duty cycle values.

\section{Appendices}

\subsection{Despread Receiver Noise Variance Derivation}

Recall that

$$
\mathbf{N}=\left[\begin{array}{c}
\mathbf{n}_{1} \\
\mathbf{n}_{2} \\
\vdots \\
\mathbf{n}_{N_{c}}
\end{array}\right]=\left[\begin{array}{cccc}
n_{11} & n_{12} & \cdots & n_{1 L} \\
n_{21} & n_{22} & \cdots & n_{2 L} \\
\vdots & \vdots & \ddots & \vdots \\
n_{N_{c} 1} & n_{N_{c} 2} & \cdots & n_{N_{c} L}
\end{array}\right]
$$

The despread row noise vector

$$
\mathbf{n}_{D}=\frac{1}{\|\mathbf{c}\|} \mathbf{c}^{\dagger} \mathbf{N}=\frac{1}{\|\mathbf{c}\|}\left[\begin{array}{c}
\sum_{a=1}^{N_{c}} c_{a}^{*} n_{a 1} \\
\sum_{a=1}^{N_{c}} c_{a}^{*} n_{a 2} \\
\vdots \\
\sum_{a=1}^{N_{c}} c_{a}^{*} n_{a L}
\end{array}\right]^{t}
$$

and it's hermitian is given by

$$
\mathbf{n}_{D}^{\dagger}=\frac{1}{\|\mathbf{c}\|} \mathbf{N}^{\dagger} \mathbf{c}=\frac{1}{\|\mathbf{c}\|}\left[\begin{array}{c}
\sum_{a=1}^{N_{c}} n_{a 1}^{*} c_{a} \\
\sum_{a=1}^{N_{c}} n_{a 2}^{*} c_{a} \\
\vdots \\
\sum_{a=1}^{N_{c}} n_{a L}^{*} c_{a}
\end{array}\right]
$$

Recalling from (19), the covariance is then

$$
\begin{aligned}
& \operatorname{cov}\left(\mathbf{n}_{D}\right)=\frac{1}{\|\mathbf{c}\|^{2}} \otimes \\
& {\left[\begin{array}{ccc}
\sum_{a=1}^{N_{c}} \sum_{b=1}^{N_{c}} \mathrm{E}\left[c_{b}^{*} n_{b 1} n_{a 1}^{*} c_{a}\right] & \ldots & \sum_{a=1}^{N_{c}} \sum_{b=1}^{N_{c}} \mathrm{E}\left[c_{b}^{*} n_{b L} n_{a 1}^{*} c_{a}\right] \\
\sum_{a=1}^{N_{c}} \sum_{b=1}^{N_{c}} \mathrm{E}\left[c_{b}^{*} n_{b 1} n_{a 2}^{*} c_{a}\right] & \ldots & \sum_{a=1}^{N_{c}} \sum_{b=1}^{N_{c}} \mathrm{E}\left[c_{b}^{*} n_{b L} n_{a 2}^{*} c_{a}\right] \\
\vdots & \ldots & \vdots \\
\sum_{a=1}^{N_{c}} \sum_{b=1}^{N_{c}} \mathrm{E}\left[c_{b}^{*} n_{b 1} n_{a L}^{*} c_{a}\right] & \cdots & \sum_{a=1}^{N_{c}} \sum_{b=1}^{N_{c}} \mathrm{E}\left[c_{b}^{*} n_{b L} n_{a L}^{*} c_{a}\right]
\end{array}\right]}
\end{aligned}
$$

Since the sampled receiver noise is AWGN, we know that

$$
\begin{aligned}
\mathrm{E}\left[n_{i j} n_{k l}^{*}\right] & =\sigma^{2}, & & i=k, \quad j=l \\
& =0, & & \text { otherwise. }
\end{aligned}
$$

Thus, (44) reduces to

$$
\begin{aligned}
& \operatorname{cov}\left(\mathbf{n}_{D}\right)= \\
& \frac{1}{\|\mathbf{c}\|^{2}}\left[\begin{array}{cccc}
\sum_{a=1}^{N_{c}}|c|^{2} \sigma^{2} & 0 & \cdots & 0 \\
0 & \sum_{a=1}^{N_{c}}|c|^{2} \sigma^{2} & \cdots & 0 \\
\vdots & \vdots & \ddots & \vdots \\
0 & 0 & \cdots & \sum_{a=1}^{N_{c}}|c|^{2} \sigma^{2}
\end{array}\right]
\end{aligned}
$$

Since $\|\mathbf{c}\|^{2}=\sum_{a=1}^{N_{c}}|c|^{2}$, then the covariance is simply

$$
\operatorname{cov}\left(\mathbf{n}_{D}\right)=\sigma_{n}^{2} \mathbf{I}_{L_{t} X L_{t}}
$$




\subsection{Simplification of Tone Interference Vector}

It can be shown that the tone vector in (30) is given by

$$
\mathbf{j}_{Q}=\frac{A_{J}}{2\|\mathbf{c}\|}\left[\begin{array}{c}
\sum_{a=1}^{N_{c}}\left(c_{I a}^{*}-j c_{Q a}^{*}\right)\left(\cos \theta_{1 a}-j \sin \theta_{1 a}\right) \\
\sum_{a=1}^{N_{c}}\left(c_{I a}^{*}-j c_{Q a}^{*}\right)\left(\cos \theta_{2 a}-j \sin \theta_{2 a}\right) \\
\vdots \\
\sum_{a=1}^{N_{c}}\left(c_{I a}^{*}-j c_{Q a}^{*}\right)\left(\cos \theta_{L a}-j \sin \theta_{L_{t} a}\right)
\end{array}\right]^{t}
$$

which can be expanded to

$$
\begin{aligned}
& \mathbf{j}_{Q}=\frac{A_{J}}{2\|\mathbf{c}\|} \otimes \\
& {\left[\begin{array}{c}
\sum_{a=1}^{N_{c}} c_{I a} \cos \theta_{1 a}-c_{Q a} \sin \theta_{1 a}-j c_{Q a} \cos \theta_{1 a}-j c_{I a} \sin \theta_{1 a} \\
\sum_{a=1}^{N_{c}} c_{I a} \cos \theta_{2 a}-c_{Q a} \sin \theta_{2 a}-j c_{Q a} \cos \theta_{2 a}-j c_{I a} \sin \theta_{2 a} \\
\vdots \\
\sum_{a=1}^{N_{c}} c_{I a} \cos \theta_{L_{t} a}-c_{Q a} \sin \theta_{L_{t} a}-j c_{Q a} \cos \theta_{L_{t} a}-j c_{I a} \sin \theta_{L_{t} a}
\end{array}\right],}
\end{aligned}
$$

where $c^{*}=c$ since the chips are real constants (either 1 or -1 ). We assume that there is an odd number of chips in the PN code where the number of 1 s outnumber -1 s by one such that $\sum_{a=1}^{N_{c}} c_{I a}=\sum_{a=1}^{N_{c}} c_{Q a}=1$. Then (49) simplifies to

$$
\mathbf{j}_{Q}=\frac{A_{J}}{2\|\mathbf{c}\|}\left(\begin{array}{c}
\left(\cos \theta_{1}-j \sin \theta_{1}\right)-\left(j \cos \theta_{11}+\sin \theta_{1}\right) \\
\left(\cos \theta_{2}-j \sin \theta_{2}\right)-\left(j \cos \theta_{2}+\sin \theta_{2}\right) \\
\vdots \\
\left(\cos \theta_{L_{t}}-j \sin \theta_{L_{t}}\right)-\left(j \cos \theta_{L_{t}}+\sin \theta_{L_{t}}\right)
\end{array}\right)^{t}
$$

where the subscript $a$ was dropped since there is only one term left in the summation. It is easily seen that this is equivalent to (31).

\section{REFERENCES}

[1] P. A. Bello, B. D. Nelin. Predetection diversity combining with selectivity fading channels, IRE Trans. Commun. Syst., vol. 10, pp. 32-42, Nov. 1962.

[2] W. C. Jakes. Microwave Mobile Communications, IEEE Press, 1974.

[3] I. Telatar. Capacity of multi-antenna Gaussian Channels, Eur. Trans. Telecommun., vol. 10, no. 6, pp. 585-595, Nov. 1999.

[4] F. Farrokhi, G. Foschini, A. Lozano, R. Valenzuela. Link-optimal space-time processing with multiple transmit and receive antennas, IEEE Commun. Lett., vol. 5, no. 3, pp. 85-87, Mar 2001.

[5] C. Chuah, D. Tse, J. Kahn, R. Valenzuela. Capacity scaling in MIMO wireless systems under correlated fading, IEEE Trans. Inf. Theory, vol. 48, no. 3, pp. 637-650, Mar 2002.

[6] P. Smith, S. Roy, and M. Shafi. Capacity of MIMO systems with semicorrelated flat fading, IEEE Trans. Inf. Theory, vol. 49 , no. 10, pp. 2781-2788, Oct 2003.

[7] Y. Wang, D. Yue. Capacity of MIMO Rayleigh fading channels in interference, IEEE Trans. Veh. Tech., vol. 58, no. 8, pp. 4398-4405, Oct 2009.

[8] E. Biglieri, G. Taricco, Transmission and Reception with Multiple Antennas: Theoretical Foundations, Now Publishers, 2004.

[9] V. Tarokh, N. Seshadri, A. R. Calderbank, Space-time codes for high data rate wireless communications: performance criterion and code construction, IEEE Trans. Inform. Theory, vol. 44, no. 2, pp. 744-765, Mar, 1998. 
[10] S. M. Alamouti, A simple transmit diversity technique for wireless communication, IEEE J. Selected Areas Comm., vol. 16, no. 8, pp. 1451-1458, 1998.

[11] E. G. Larsson, P. Stoica. Space-Time Block Coding for Wireless Communications, Cambridge: Cambridge University Press, 2003.

[12] A. Paulraj, R. Nabar, D. Gore. Introduction to Space-Time Wireless Communications, Cambridge: Cambridge University Press, 2003.

[13] E. Biglieri, et al. MIMO Wireless Communications, Cambridge: Cambridge University Press, 2003.

[14] B. Vucetic, J. Yuan. Space-Time Coding, John Wiley and Sons, Ltd, West Sussex, England, 2003.

[15] R. A. Scholtz. The origins of spread spectrum communications, IEEE Trans. Commun., vol. 30, no. 5, pp. 822-852, Nov. 1982

[16] S. W. Golomb. Shift Register Sequences, San Francisco, CA, Holden-Day, 1967.

[17] M. K. Simon, J. K. Omura, R. A Scholtz, and B. K. Levitt. Spread Spectrum Communications, Rockville, MD , Computer Science Press, 1985.

[18] D. J. Torrieri. Principles of Secure Communications, London, UK, Artech House, 1985.

[19] D. V. Sarwate, M. B. Pursely. Crosscorellation properties of pseudorandom and related sequences, Proc. IEEE, vol. 68, no. 5, pp. 593-619, Nov. 1980.

[20] R. C. Dixon. Spread Spectrum Systems with Commercial Applications, 3rd ed, Chichester: John Wiley \& Sons, Artech House, 1994.

[21] R. Peterson, R. Ziemer, D. Borth. Introduction to Spread-Spectrum Communications, Prentice Hall, Englewood Cliffs, NJ, 1995.

[22] A. J. Viterbi. CDMA: Principles of Spread Spectrum Communications, Reading, MA, Addison-Wesley, 1995.

[23] J. S. Lee, L. E. Miller. CDMA Systems Engineering Handbook, London, Artech House, 1998.

[24] J. Wang, and J. Chen. Performance of Widebanc CDMA Systems with Complex Spreading and Imperfect Channel Estimation, IEEE Journal on Selected Areas in Communications, vol. 19, no. 1, pp. 152-163, Jan. 2001.

[25] T. T. Ha. Theory and Design of Digital Communication Systems, Cambridge University Press, UK, 2011.

[26] T. Macdonald, M. Pursely. Complex processing in quaternary direct-sequence spread-spectrum receivers, IEEE Journal on Selected Areas in Communications, vol. 19, pp. 494-498, Oct. 1998.

[27] T. Macdonald, and M. Pursely. The performance of direct-sequence spread spectrum with complex processing and quartenary data modulation, IEEE Journal on Selected Areas in Communications, vol. 18, pp. 1408-1417, Aug, 2000.

[28] E. Mintzias, R. A. Romero, T. Ha. Performance Evaluation of Complex Spreading MIMO Systems in Rayleigh Fading and Interference," IEEE Int Waveform Diversity and Design Conf, Jan, 2012.

[29] D. G. Brennan. Linear diversity combining techniques, Proc. IRE, vol. 46, pp. 1075-1102, Jun, 1959.

[30] M. Z. Win, Z. A. Kostic. Impact of spreading bandwidth on RAKE reception in dense multipath channels, IEEE Journal on Selected Areas in Communications, vol. 17, pp. 1794-1806, 1999. 
[31] M. J. Gans. The effect of Gaussian errors in maximal ratio combiners, IEEE Trans. Commun. Tech., vol. 19, pp. 492-500, Aug, 1971.

[32] A. Goldsmith. Wireless Communications, Cambridge University Press, UK, 2005. 